Elsevier required licence: (C) $<2015>$. This manuscript version is made available under the CC-BY-NC-ND 4.0 license http://creativecommons.org/licenses/by-nc-nd/4.0/ 


\title{
Removal of oil from water using magnetic bicomponent composite nanofibers fabricated by electrospinning
}

Zhe Jiang ${ }^{1}$, Leonard D. Tijing ${ }^{2}$, , Altangerel Amarjargal ${ }^{3}$, Chan Hee Park ${ }^{1,4, *}$, Kyoung-Jin $\mathrm{An}^{5}$, Ho Kyong Shon ${ }^{2}$, and Cheol Sang Kim ${ }^{1,4} *$

${ }^{1}$ Department of Bionanosystem Engineering, Graduate School, Chonbuk National University, Jeonju, Jeonbuk 561-756, Republic of Korea

${ }^{2}$ Centre for Technology in Water and Wastewater, School of Civil and Environmental Engineering, Faculty of Engineering and Information Technology, University of Technology, Sydney (UTS), 15 Broadway, NSW 2007, Australia

${ }^{3}$ Power Engineering School, Mongolian University of Science and Technology, Ulaanbaatar, Mongolia

${ }^{4}$ Division of Mechanical Design Engineering, Chonbuk National University, Jeonju, Jeonbuk 561-756, Republic of Korea

${ }^{5}$ School of Energy and Environment, City University of Hong Kong, Tat Chee Avenue, Kowloon, Hong Kong SAR

*Corresponding authors: L.D. Tijing: E-mail: 1tijing@gmail.com; leonard.tijing@uts.edu.au; C.H. Park, biochan@jbnu.ac.kr, and; C.S. Kim, Fax: +82 63270 2460, E-mail: chskim@jbnu.ac.kr

\begin{abstract}
In the present study, a magnetic nanofibrous composite mat composed of polystyrene (PS)/polyvinylidene fluoride (PVDF) nanofibers with selective incorporation of iron oxide $\left(\mathrm{Fe}_{3} \mathrm{O}_{4}\right)$ nanoparticles (NPs) on/in PS was successfully prepared via a facile two-nozzle electrospinning process for oil-in-water separation. Field emission scanning electron microscopy and infrared spectroscopy showed the mats to be highly-porous in structure and confirmed the presence of the $\mathrm{Fe}_{3} \mathrm{O}_{4}$ NPs on/in the nanofibers. Both PS and PVDF nanofibers exhibited oleophilic and hydrophobic properties. The results showed improved mechanical properties when PVDF was added to the composite mat compared to the pristine PS mat. In addition, the incorporation of magnetic $\mathrm{Fe}_{3} \mathrm{O}_{4} \mathrm{NPs}$ in the composite mat helps in the easy recovery of the mats after the oil-in-water sorption process. The composite mats showed good oil sorption capacity (35-46 g/g) and improved mechanical property. The present electrospun magnetic PVDF/ $/ \mathrm{Fe}_{3} \mathrm{O}_{4} @ \mathrm{PS}$ nanofibers could be potentially useful for the efficient removal of oil in water and recovery of sorbent material.
\end{abstract}

Keywords: A. Polymer fiber; A. Nano-structures; B. Adhesion; E. Electrospinning

\section{Introduction}

The issue on oil pollution has drawn continuous attention of the society in recent years. Oil spills are mainly caused by shipping accidents, offshore or marine vessel leakage and illegal 
discharges of oily wastes [1], which consequently endanger the ecosystem and marine lives, and contaminate beaches and shorelines [2]. Additionally, domestic and industrial wastes which contain excess oil or fuel and drained in sewers or carried in waterways are also sources of pollution [3]. These oil pollution problems have prompted a necessity to develop a cost-effective and environmentfriendly way of oil spill cleanup. Among the commonly used methods include in situ burning of oil on water [4], mechanical extraction [5], chemical degradation [6], and centrifugation and gravity separation. Each of these methods has its own limitation. In most cases, the cleanup requires the use of several techniques together. The common potential risk is the secondary pollution from the cleanup such as the generation of smoke and dust from the in situ burning of oil on water and contamination by toxic compounds from the use of commercial chemical dispersants [7]. Furthermore, these oil cleanup methods have low separation efficiency, complex equipment, and high operation cost.

Among the cleanup methods, the use of sorbent materials as a mechanical extraction method is considered to be a promising technique. Sorbents are very commonly used during oil spills for their cost-effectiveness and affordability [8]. These sorbents include natural materials, inorganic mineral products and organic synthetic fibers. An ideal sorbent material should have high hydrophobicity, high oleophilicity, high uptake capacity and rate, adequate buoyancy, and good recoverability of the adsorbed oil [9]. Recently, considerable research on natural organic sorbents was carried out such as the use of Kapok fiber [10], cotton, and rice husks [11]. A recent study reported on the coating of graphene on cotton for oil/water separation [12]. Even though natural organic sorbents are mostly biodegradable, some of them absorb both water and oil thus lessening the separation efficiency, and some of them may also sink during absorption with harsh sea condition [13]. Mineral products suffer from low buoyancy and low oil sorption capacity.

On the other hand, synthetic sorbents are man-made materials designed to combat oil spillages. Due to their excellent (super/) hydrophobic and (super/) oleophilic properties, they normally achieve higher sorption capacity [14]. The most widely used synthetic sorbents consisted of high molecular weight polymers, such as polyurethane (PU), polyethylene (PE), or polypropylene (PP) [15]. Nonwoven PP fibrous mats are widely used for oil spill cleanups due to their good oleophilichydrophobic properties, adequate buoyancy, and their scalable production. However, nonwoven PP fibers have low oil-sorption capacity. As synthetic fibers are mostly non-biodegradable, the full recovery of the sorbent after adsorption is very important to avoid secondary pollution. Sorbents could sink and are sometimes hard to recover after adsorption due to many factors including environment conditions such as wind, current, tides, etc. Providing a magnetic property on the sorbents can be useful in the recovery process from the water surface. Chen et al. [16] and Adriana et al. [17] fabricated different oil sorbents both providing magnetic properties to achieve the idea of easy re-pick after the sorbents are saturated. Wei et al. used a biosurfactant to clean used oil PP sorbents and more than $95 \%$ removal of oil from the sorbents was achieved with certain washing conditions [18]. Other reports have also indicated the potential for recycling the used sorbent on other synthetic sorbents. 
The $\beta$-cyclodextrin oil-absorbents made by Ding et al. [19] were reusable. The oil can be desorbed by a chemical extraction method [19].

With the advances in science and technology, it is now possible to fabricate materials at the nanoscale level. Electrospinning is one of the well-known techniques in the fabrication of polymeric fibers with ultrafine diameters from a polymeric solution or melt [20,21]. The resulting micro/nanofibers are formed in a nonwoven structure, which possess high porosity, interconnected pores, high surface area-to-volume ratio, and high strength-to-weight ratio [22, 23]. Depending on the desired morphology and properties, the materials and process parameters for electrospinning can be manipulated. Solid or internally-porous fibers, or smooth or rough fiber surface can be obtained by just a simple manipulation of several parameters. Composite mats can also be fabricated by incorporating nanoparticles through simple blending with the polymer solution to provide additional functionalities [24], or the combination of different polymeric nanofibers into one hybrid mat through multi-nozzle electrospinning [25]. Multi-nozzle electrospinning combines the properties of different polymers to provide a synergistic property effect on the hybrid mat [26]. Several studies have reported promising results on the use of electrospun nanofibers for oil-sorption [27] and other applications [28$31]$.

Polysterene (PS) nanofibers have shown good oleophilicity and hydrophobicity, and good oil sorption capacity [32]. But it is extremely light weight and has a cotton-like appearance, which makes it tend to flow and drift in air. In addition, the poor mechanical properties of PS fiber mats might cause problems during transportation and pick-up after oil absorption in the open water. Hence, one way to improve the mechanical properties of PS nanofibers is to combine it with other material forming a composite while utilizing its oil-sorption potential. In this study, we utilize the good properties of polyvinylidene fluoride (PVDF) to add mechanical stability to the PVDF/PS membrane. PVDF is widely applied in filtration systems as membranes [33, 34]. Electrospun PVDF fibers are hydrophobic, have high chemical resistance and good mechanical strength, excellent thermal stability, and steady performance for long-term application [33]. The addition of PVDF nanofibers could provide increased mechanical properties of the composite mat. Additionally, to aid in the better recovery of the sorbent material, the provision of magnetic properties in the composite mat would be a good strategy. Among the available nanoparticles, magnetic iron oxide $\left(\mathrm{Fe}_{3} \mathrm{O}_{4} / \gamma \mathrm{Fe}_{3} \mathrm{O}_{4}\right)$ are proven to have low degree of cytotoxicity even at high concentrations [35], thus its possible use in oil-sorption sorbents would be viable. Magnetite $\left(\mathrm{Fe}_{3} \mathrm{O}_{4}\right)$ nanoparticles have been studied for use in water treatment where they show promising ability in removing heavy metal ions with high efficiency [36]. The magnetic properties of $\mathrm{Fe}_{3} \mathrm{O}_{4}$ nanoparticles and their proper dispersion in the composite polymeric materials would provide magnetic properties for easy recovery of the sorbent material.

In this study, we prepared and fabricated a nanocomposite sorption membrane consisting of two different polymeric components: PS and PVDF, which were simultaneously fabricated into one mat by one-step two-nozzle electrospinning. The PS component nanofibers were selectively 
incorporated with $\mathrm{Fe}_{3} \mathrm{O}_{4}$ NPs to provide magnetic properties. PVDF nanofibers serve as a support for the mechanical integrity of the composite membrane. The objective of this study was to study the effect of both PVDF nanofibers and $\mathrm{Fe}_{3} \mathrm{O}_{4}$ NPs on the mechanical properties and sorption efficiency of the PS-based composite membranes. We investigated the oil sorption capacities of the fabricated composite membranes as well as evaluated their different physical and chemical properties through various characterization methods and measurements.

\section{Experimental}

\subsection{Materials}

Polystyrene (PS, Mw = 250,000 g/mol) and polyvinylidene fluoride (PVDF, $\mathrm{Mw}=180,000$ $\mathrm{g} / \mathrm{mol}$ ) were purchased from Arcos and Sigma-Aldrich, respectively. N,N-dimethylformamide (DMF, 99.5\%), tetrahydrofuran (THE, $99.5 \%$ ), and acetone (99.5\%) were bought from Samchun Chemical, Korea. The present magnetic $\mathrm{Fe}_{3} \mathrm{O}_{4}$ nanoparticles (NPs) (Alfa Aesar) showed an average diameter of $200 \mathrm{~nm}$ from scanning electron microscopy (SEM) observation (result not shown). Four types of oil were used during the experiments: sunflower oil from Rio Santo, soybean oil from Beksul, motor oil (5W-30) and diesel oil (5W-40) from Mobil 1. Table 1 shows the viscosity and density of the oils measured at room temperature. All of the materials were used as received without further treatment.

Table 1. Properties of the oils used in the present study.

\begin{tabular}{|l|c|c|}
\hline \multicolumn{1}{|c|}{ Type of oil } & Viscosity $(\mathrm{cP})$ & Density $\left(\mathrm{g} / \mathrm{cm}^{3}\right)$ \\
\hline Sunflower oil & 64.55 & 0.874 \\
\hline Soybean oil & 61.4 & 0.855 \\
\hline Motor oil & 142.2 & 0.806 \\
\hline Diesel oil & 214.73 & 0.821 \\
\hline
\end{tabular}

\subsection{Electrospinning}

PVDF solution (20 wt $\%$ ) in DMF/acetone (7/3 ratio) mixed solvent and PS solution (20 wt $\%$ ) in DMF/THF mixed solvent (3/1 ratio) were prepared by overnight stirring at $55^{\circ} \mathrm{C}$ and at room temperature, respectively. $\mathrm{Fe}_{3} \mathrm{O}_{4}$ nanoparticles ( $5 \mathrm{wt} \%$ w.r.t. the weight of PS) were added in PS solution and the solution was subjected to bath sonication for $2 \mathrm{~h}$ to disperse the nanoparticles. Electrospinning was carried out using the set-up used in our previous study (see Fig. 1) [26]. Two different polymer solutions were simultaneously electrospun from two nozzles configured side-byside with each other oriented at an angle of $80^{\circ}$ between them. The two nozzles kept on oscillating horizontally through a step motor. Fibers were electrospun at an applied voltage of $18 \mathrm{kV}$ for all solutions at a tip-to-collector distance of $15 \mathrm{~cm}$. The feed rate was maintained at $0.8 \mathrm{ml} / \mathrm{h}$ for the PVDF solution, and $1 \mathrm{ml} / \mathrm{h}$ for all other solutions. To fabricate single component nanofibrous mats, a 
single nozzle was used, which was perpendicularly oriented to the collector. All the fiber mats were dried in an oven at $60^{\circ} \mathrm{C}$ overnight to remove the residual solvents. The fiber mats were used as sorbents with no further treatment. Table 2 shows the electrospinning parameters and the corresponding sample names of different fabricated membranes to aid in the discussion.

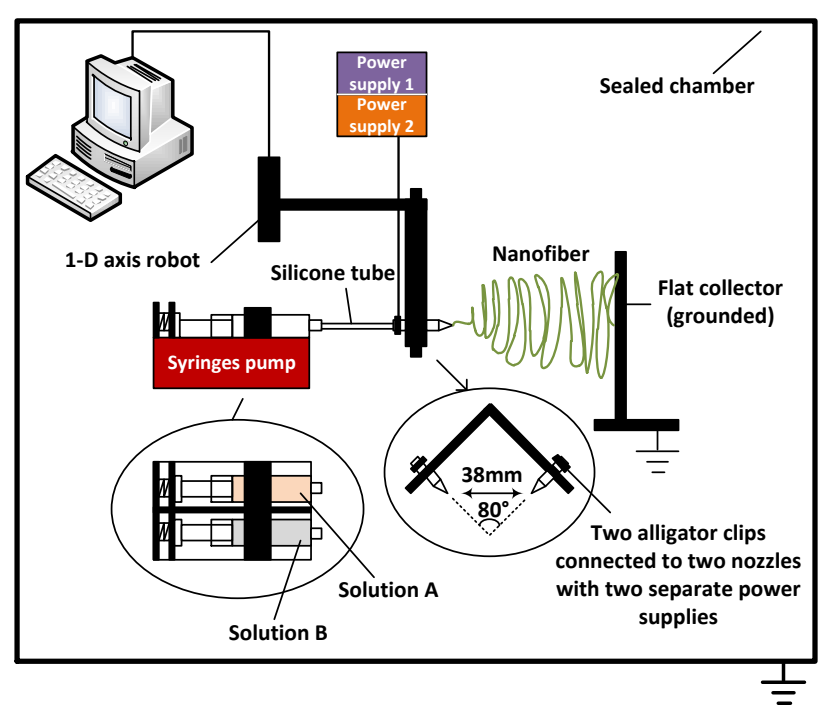

Fig. 1. Schematic illustration of the present multi-nozzle electrospinning set-up.

Table 2. Electrospinning parameters and the corresponding names of the fabricated mats.

\begin{tabular}{|c|c|c|c|c|c|}
\hline Sample & Method & \multicolumn{3}{|c|}{ Feed rate } & $\begin{array}{c}\text { Electrospun } \\
\text { volume }\end{array}$ \\
\hline PVDF & \multirow{3}{*}{ One-nozzle } & \multicolumn{3}{|c|}{$0.8 \mathrm{ml} / \mathrm{h}$} & $4 \mathrm{ml}$ \\
\hline PS & & \multicolumn{3}{|c|}{$1 \mathrm{ml} / \mathrm{h}$} & $4 \mathrm{ml}$ \\
\hline $\mathrm{Fe}_{3} \mathrm{O}_{4} @ \mathrm{PS}$ & & \multicolumn{3}{|c|}{$1 \mathrm{ml} / \mathrm{h}$} & $4 \mathrm{ml}$ \\
\hline \multirow{2}{*}{ PVDF/PS } & \multirow{2}{*}{ Two-nozzle } & A & PVDF & $0.8 \mathrm{ml} / \mathrm{h}$ & $1.8 \mathrm{ml}$ \\
\hline & & B & PS & $1 \mathrm{ml} / \mathrm{h}$ & $2.25 \mathrm{ml}$ \\
\hline \multirow[t]{2}{*}{$\mathrm{PVDF} / \mathrm{Fe}_{3} \mathrm{O}_{4} @ \mathrm{PS}$} & \multirow{2}{*}{ Two-nozzle } & A & PVDF & $0.8 \mathrm{ml} / \mathrm{h}$ & $1.8 \mathrm{ml}$ \\
\hline & & B & $\mathrm{Fe}_{3} \mathrm{O}_{4} @ \mathrm{PS}$ & $1 \mathrm{ml} / \mathrm{h}$ & $2.25 \mathrm{ml}$ \\
\hline
\end{tabular}

\subsection{Measurement of oil sorption capacity}

The tests for oil sorption capacity were carried out following ASTM F726-60 standard method using four different kinds of oil [14, 37]. Briefly, in a $200 \mathrm{ml}$ glass bottle, $10 \mathrm{~g}$ oil was added into $150 \mathrm{ml}$ water. A thin oil layer was formed on the water immediately because of the lower density of oil compared to water. The sorbent was then gently placed on the oil/water mixture. The wet sorbent was retrieved using tweezers and then drained by hanging for $3 \mathrm{~min}$ in air to remove the excess oil before weighing. The oil sorption capacity is quantified by the weight ratio as oil absorbency $(\mathrm{g} / \mathrm{g})$ from the following equation: 


$$
\mathrm{Q}_{\mathrm{oil}}=\frac{m_{s}-m_{i}}{m_{i}}
$$

where $\mathrm{Q}_{\text {oil }}$ is the oil sorption capacity ( $\mathrm{g} / \mathrm{g}$ ) of the sample, $m_{S}$ is the weight of the wet sorbent after draining $(\mathrm{g})$, and $m_{i}$ is the initial weight of the dried sorbent before sorption $(\mathrm{g})$. Each type of sample was measured three times.

\subsection{Characterization and measurements}

The surface morphology of electrospun fibers were observed by field emission scanning electron microscopy (FE-SEM) (Hitachi S-4800, Japan) and transmission electron microscopy (TEM) (H-7650, Hitachi, Japan). The samples for FE-SEM were sputtered with platinum (Pt) via a $\mathrm{K} 575 \mathrm{x}$ coater (Emitech, UK). An energy dispersive X-ray spectroscopy (EDS) attached to FE-SEM was used to analyse the surface chemical content of the samples. The samples for TEM were prepared by directly electrospinning on a copper grid mesh for 5-10 sec. The fiber diameter distribution was processed using ImageJ software (NIH, USA). At least 50 fibers were measured for each sample based from SEM images. The material analysis was done by Fourier transform infrared spectroscopy (FT-IR) via Paragon 1000 Spectrometer (PerkinElmer, USA) in the range of $400-4000 \mathrm{~cm}^{-1}$ with a signal resolution of $1 \mathrm{~cm}^{-1}$ and a minimum of 16 scans. Contact angle was measured by Digidrop Goniometer (GBX, France). During each contact angle test, a drop of deionized water $(\mathrm{d}=6 \mathrm{~mm})$ was dropped onto the fiber mat automatically. Each sample was tested 3 times and the average contact angle was calculated. The mechanical properties were tested using a bench-type Lloyd LR5KPlus testing machine (AMETEK, Inc. USA) with a load limit of $100 \mathrm{~N}$ according to ASTM D882-10. The crosshead speed was $5 \mathrm{~mm} / \mathrm{min}$. Dog bone specimens (gage width $=10 \mathrm{~mm}$, sample length $=70 \mathrm{~mm}$ ) were prepared, which were fixed on a paper frame by taping both ends of the specimen. At least 5 measurements were tested for each sample and the average value is reported here. The thickness of the specimens was measured by a microcaliper (Mitutoyo Corp., Japan).

\section{Results and discussion}

\subsection{Physico-chemical characteristics of the fabricated mats}

Electrospinning is a facile technique to fabricate porous-structured fiber mats with ultrafine fibers, small pore sizes and high surface area [38] through an electrically-charged jet of polymer solution or melt [33]. The morphology and structure of electrospun fibers can be manipulated by adjusting the materials and process parameters. Electrospinning results into three typical morphologies: beads, bead-on-string and smooth or rough fibers [39] depending on the process and material parameters. Figure 2 shows the FE-SEM images of the present neat and composite fibers. All fibers showed highly porous and non-woven structure with very minimal bead-on-string formation. The formation of beads on the fibers is to be avoided because they can serve as stress points that could weaken the mechanical properties of the mat. Additionally, beads can cause non- 
uniformity in the mat, which can affect the oil sorption performance. The neat PVDF nanofibers showed smooth surfaces and solid cylindrical shape with diameter in the range of $20-100 \mathrm{~nm}$ (Fig. 2a), forming a Gaussian distribution. On the other hand, neat PS fibers had diameters ranging from $700 \mathrm{~nm}$ to $1.6 \mu \mathrm{m}$ (Fig. 2b), which were a few orders of magnitude bigger than PVDF nanofibers. PS fibers showed rough surface morphology with long continuous cracks along the axis of the fiber. This kind of PS morphology increases the overall surface area, which could benefit the oil-sorption capacity of the membrane. Meanwhile, the $\mathrm{Fe}_{3} \mathrm{O}_{4} / \mathrm{PS}$ composite fibers showed similar rough morphology but with decreased fiber diameter sizes, ranging from $500 \mathrm{~nm}$ to $1.4 \mu \mathrm{m}$ (Fig. 2c), which is similar with the observation of other studies [38]. This is attributed to the increased conductivity of the PS solution when $\mathrm{Fe}_{3} \mathrm{O}_{4}$ NPs were incorporated, leading to an increased charge carrying ability, thus elongating the fiber more as it is subjected to the electric field resulting to thinner fibers [38]. The bicomponent composite mats, i.e., PS/PVDF with or without $\mathrm{Fe}_{3} \mathrm{O}_{4}$ NPs showed some beads-on-string formation (Fig. 2d). Beads are reported to help in increasing the hydrophobic property of a surface by providing a rougher surface as observed by other studies [40]. Fig. 2e clearly shows the successful incorporation of $\mathrm{Fe}_{3} \mathrm{O}_{4}$ NPs fully encapsulated by the PS fibers. Proper dispersion of nanoparticles in the polymer matrix is important to ensure good load transfer from the polymer to the nanoparticles, thereby helping improve the mechanical properties. Here, $\mathrm{Fe}_{3} \mathrm{O}_{4} \mathrm{NPs}$ were found to be widely distributed throughout the composite mat, thus ensuring a good magnetic property in the overall mat.
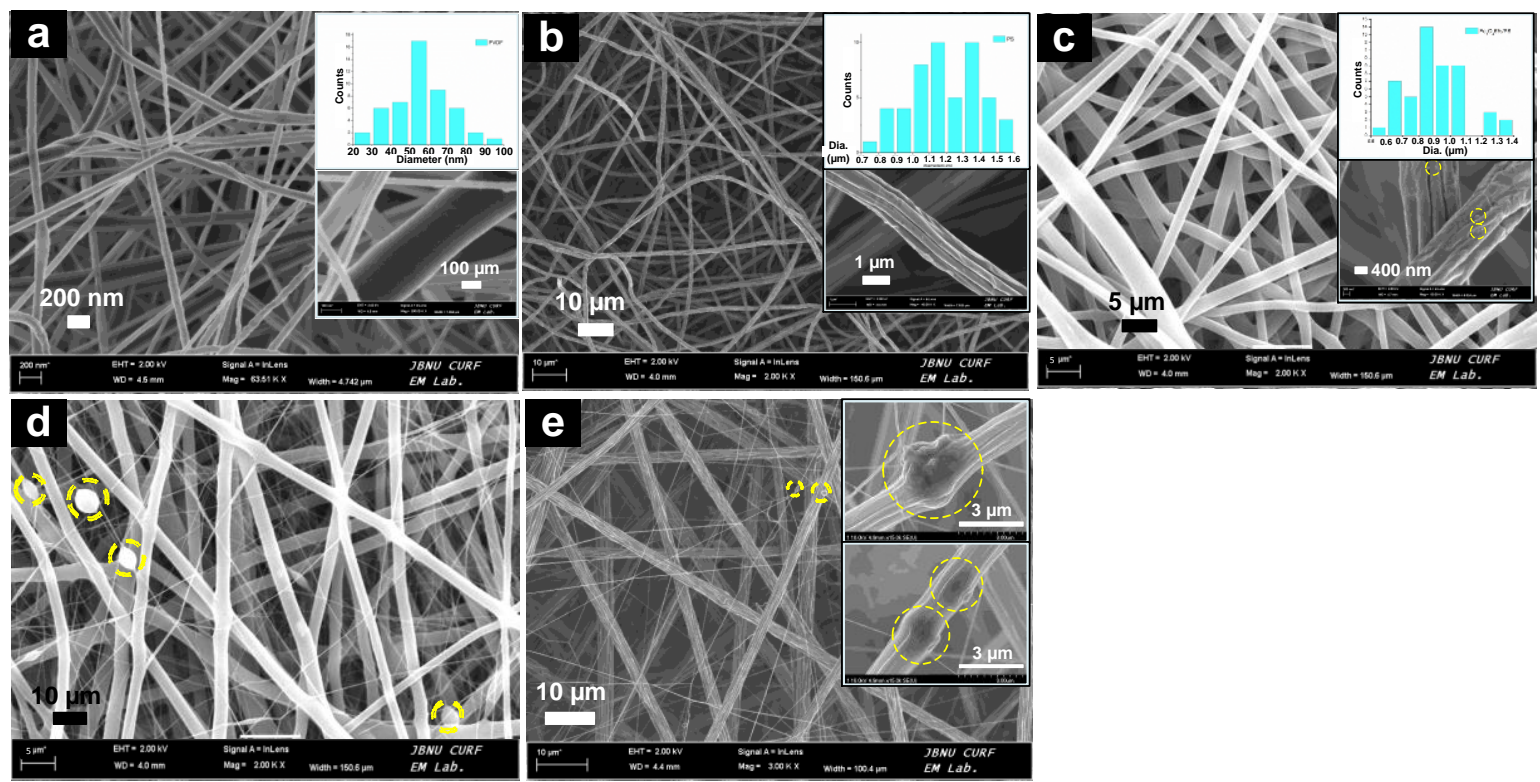

Fig. 2. FE-SEM images and corresponding fiber distributions (insets) of the electrospun fibers and composites: (a) PVDF, (b) PS. (c) $\mathrm{Fe}_{3} \mathrm{O}_{4} @ P S$, (d) PVDF/PS, and (e) PVDF/Fe $\mathrm{F}_{3} @$ @PS. The single dotted circles indicate the presence of $\mathrm{Fe}_{3} \mathrm{O}_{4}$ NPs. The double dotted circles indicate the presence of beads on string.

The optical images of the fabricated mats are shown in Fig. 3. The neat PVDF mat (Fig. 3a) appears to be flat with a typical thin film-like structure. However, the neat PS mat (Fig. 3b) was very 
fluffy and cotton-like in structure, and tends to flow and drift in air easily. A darker colored mat was obtained when $\mathrm{Fe}_{2} \mathrm{O}_{3}$ NPs were incorporated into PS nanofiber (Fig. 3c). However, when both PS and PVDF were electrospun simultaneously by two-nozzle electrospinning, a less fluffy and less flat (somewhere in between the structures of the individual components) structure was observed (Fig. 3d). The incorporation of $\mathrm{Fe}_{3} \mathrm{O}_{4} \mathrm{NPs}$ has resulted to a darker color (Fig. 3e) compared to mats without them (white color) indicating that $\mathrm{Fe}_{3} \mathrm{O}_{4} \mathrm{NPs}$ are present in the mat. By using an external magnet, the composite mat containing $\mathrm{Fe}_{3} \mathrm{O}_{4}$ NPs was attracted and attached well to the magnet as shown in Fig. 3f, which confirms the successful incorporation of $\mathrm{Fe}_{3} \mathrm{O}_{4} \mathrm{NPs}$.

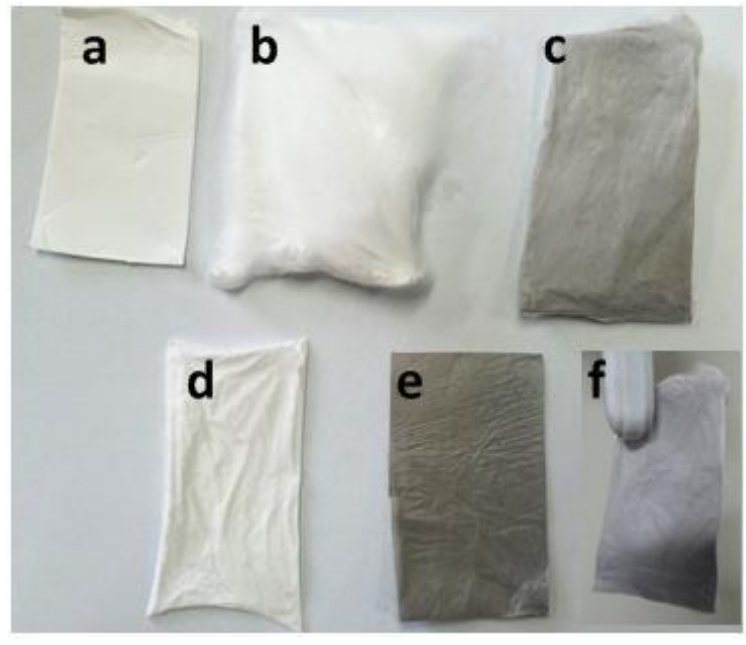

Fig. 3. Optical images of the fabricated mats: (a)PVDF, (b)PS. (c) $\mathrm{Fe}_{3} \mathrm{O}_{4} @ P S$, (d)PVDF/PS, (e) $\mathrm{PVDF} / \mathrm{Fe}_{3} \mathrm{O}_{4} @ \mathrm{PS}$, and (f) magnetic composite mat when exposed to an external magnet.

To further confirm the loading of $\mathrm{Fe}_{3} \mathrm{O}_{4} \mathrm{NPs}$ in/on the PS fibers, fibers incorporated with $\mathrm{Fe}_{3} \mathrm{O}_{4} \mathrm{NPs}$ were directly electrospun onto a copper grid mesh and were subjected to TEM. Figure 4 shows the TEM images of $\mathrm{Fe}_{3} \mathrm{O}_{4} / \mathrm{PS}$ fibers. Several images from different fiber samples were taken, and it has been observed that the $\mathrm{Fe}_{3} \mathrm{O}_{4}$ nanoparticles were mostly dispersed inside the fiber (Figs. 4a and 4b) (i.e., being fully encapsulated by the polymer) at a high concentration. But other parts also showed the $\mathrm{Fe}_{3} \mathrm{O}_{4} \mathrm{NPs}$ to be protruding on the surface of the fibers (Fig. 4c), where the lower part of the $\mathrm{Fe}_{3} \mathrm{O}_{4} \mathrm{NPs}$ is firmly attached to the fibers. It is essential for NPs to be strongly adhered on/in the fibers to prevent them from being washed away during the oil collection process and avoid any secondary contamination. Our present composite material showed firmly attached NPs on/in the fibrous mat. Additionally, the EDS measurement (result not shown) shows some peaks of Fe, which confirms the presence of $\mathrm{Fe}_{3} \mathrm{O}_{4}$ nanoparticles on/in the fibers. It is believed that with the incorporation of magnetic NPs, it will give more advantage for recovery of the composite mat after sorption through the use of an external magnet. 

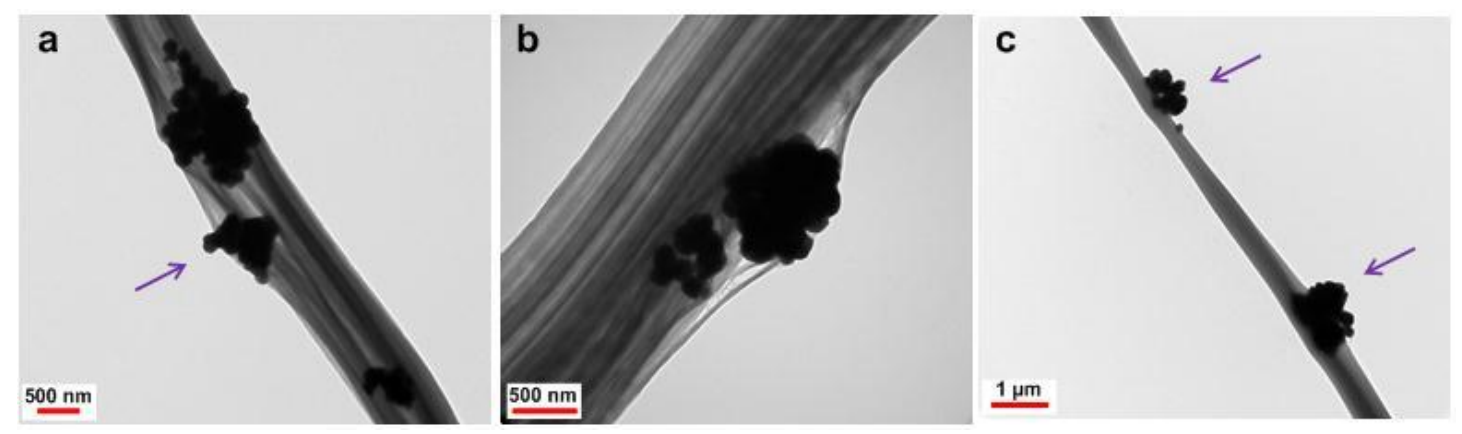

Fig. 4. TEM images of electrospun $\mathrm{Fe}_{3} \mathrm{O}_{4} @ \mathrm{PS}$ nanofiber at different locations of the nanofiber mat.

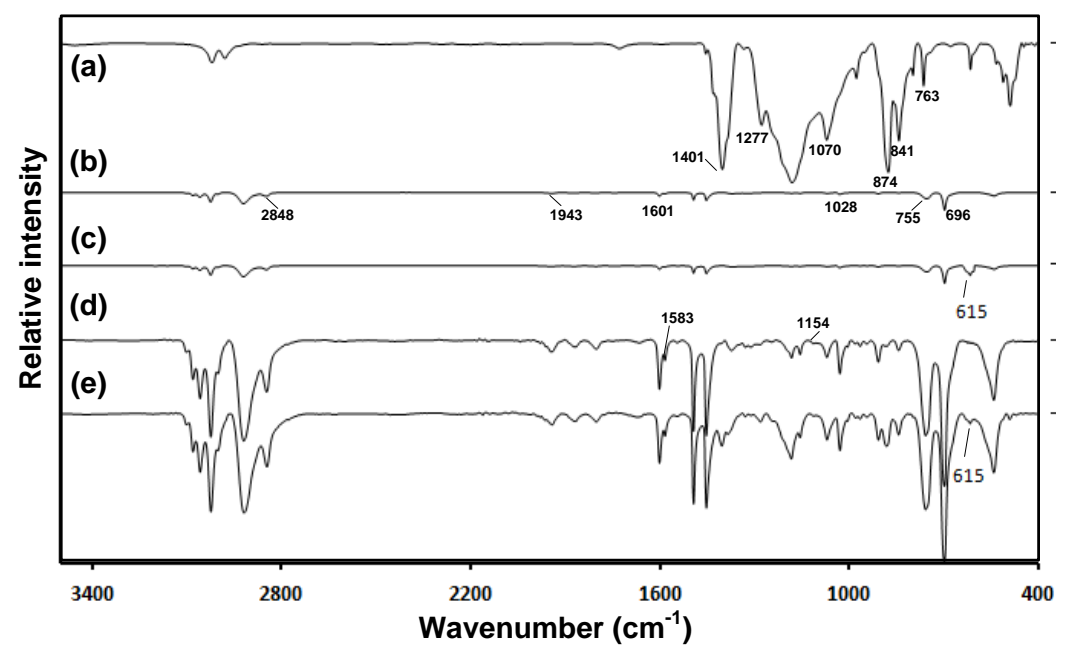

Fig. 5. FT-IR spectra of different fabricated mats: (a) PVDF, (b) PS, (c) $\mathrm{Fe}_{3} \mathrm{O}_{4} / \mathrm{PS}$, (d) PVDF-PS, and (e) $\mathrm{PVDF}-\mathrm{Fe}_{3} \mathrm{O}_{4} / \mathrm{PS}$.

Figure 5 shows the FT-IR spectra of the neat and composite nanofibrous mats. Several characteristic peaks of PVDF can be observed in the neat PVDF mat (Fig. 5a) indicating the deformation vibration band of $\mathrm{CH}_{2}$ at $1401 \mathrm{~cm}^{-1}$, the stretching band of $\mathrm{C}-\mathrm{C}$ in $\beta$-phase at $1070 \mathrm{~cm}^{-1}$, the framework vibration of $\mathrm{C}-\mathrm{C}$ at $874 \mathrm{~cm}^{-1}$, and the vibration absorption for crystalline phase at 763 $\mathrm{cm}^{-1}$ [41]. The intense peaks at $841 \mathrm{~cm}^{-1}$ and $1277 \mathrm{~cm}^{-1}$ indicate the characteristic bands of $\beta$-phase PVDF crystallites [42]. In Fig. 5b, the bands at $755 \mathrm{~cm}^{-1}$ and $696 \mathrm{~cm}^{-1}$ (bending mono-substituted benzene of C-H ), $2848 \mathrm{~cm}^{-1}$ (the stretching vibration absorption of saturated C-H), $1943 \mathrm{~cm}^{-1}$ (aromatic combination frequency overtones of C-H), $1601 \mathrm{~cm}^{-1}$ and $1583 \mathrm{~cm}^{-1}$ (aromatic bonds stretching vibration of C-C), $1154 \mathrm{~cm}^{-1}$ and $1028 \mathrm{~cm}^{-1}$ (aromatic deformation vibration of $\mathrm{C}-\mathrm{H}$ ) correspond to the chemical structure of PS. In Figs. $\mathbf{5 c}$ and $\mathbf{5 e}$, new peaks at $615 \mathrm{~cm}^{-1}$ and $620 \mathrm{~cm}^{-1}$ are observed, which are assigned to the Fe-O stretching modes of the magnetic lattice [43], indicating the successful incorporation of $\mathrm{Fe}_{3} \mathrm{O}_{4} \mathrm{NPs}$ in/on the nanofibers. Both peaks of PS and PVDF can be observed for the composite membranes (Figs. 5d and e) suggesting the presence of two component nanofibers in the composite. 


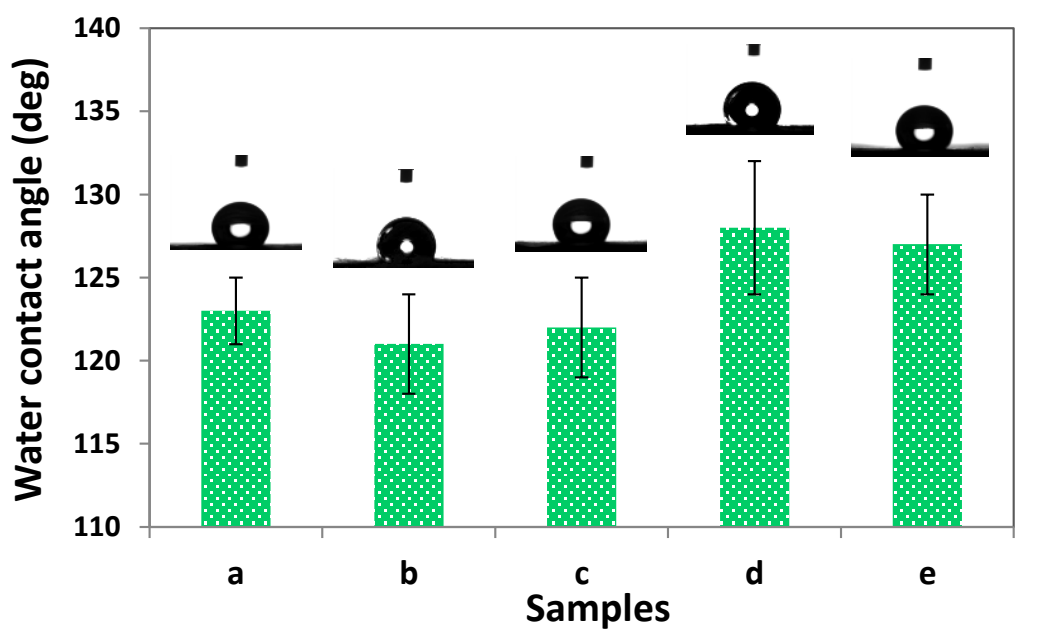

Fig. 6. Contact angles of different fabricated mats: (a) PVDF, (b) PS, (c) $\mathrm{Fe}_{3} \mathrm{O}_{4} / \mathrm{PS}$, (d) PVDF-PS, and (e) $\mathrm{PVDF}-\mathrm{Fe}_{3} \mathrm{O}_{4} / \mathrm{PS}$.

Contact angle (CA) is a quantitative measure of the wettability of a surface and it varies according to the surface energy and the solid surface roughness [44]. The average result of contact angle is given in Fig. 6. We can see that all the samples appear as hydrophobic fibers with contact angle larger than $121^{\circ}$. A slight increase in CA was observed when $\mathrm{Fe}_{3} \mathrm{O}_{4} \mathrm{NPs}$ were incorporated in PS, which may be attributed to the increased roughness due to bumps and beads formed due to $\mathrm{Fe}_{3} \mathrm{O}_{4}$ NPs (Fig. 6c). This is consistent with the studies on electrospun inorganic fibers which exhibit hydrophobic property [40]. The changes in CA could be possibly due to composition change of the membrane and the slight changing of the surface structure [45]. The nanofibers, which overlap with each other and are randomly distributed create locus for air pockets, which sustain the water droplets [46] leading to increased CA.

\subsection{Mechanical properties}

For efficient recovery, the sorbents should possess a fairly good mechanical property to withstand compression of sorbents [47]. The typical stress-strain curves and the average tensile property values are shown in Figs. 7a and 7b, respectively. In Fig. 7a, PVDF nanofibers showed a steep linear increase in the first $100 \%$ strain, and then reached a plateau until failure. Neat PS fibers showed a sudden steep increase then experienced a plastic behaviour and slow extension mode until failure. Similar trend was observed when iron oxide NPs were incorporated to PS but obtained a higher maximum tensile strength. The composite mats showed tensile strength values in between the tensile strengths of the individual components, i.e., PVDF and PS. However, the incorporation of $\mathrm{Fe}_{3} \mathrm{O}_{4}$ NPs in the composite mat has resulted to a slightly lower tensile strength, but has shown the largest elongation from among the fabricated mats. The tensile strength of PVDF nanofibrous mat was 
3.58 MPa, while the PS mat was $0.79 \mathrm{MPa}$. The composite mats fabricated by two nozzle electrospinning process have balanced the strength of the individual components, which still provide good mechanical properties as a sorbent. The tensile strength of PVDF/PS mat was $2.08 \mathrm{MPa}$, for an increase of $163.3 \%$ when compared to neat PS mat (see Fig. 7b). Additionally, the existence of $\mathrm{Fe}_{3} \mathrm{O}_{4}$ NPs within and on the surface of the PS fibers did not significantly affect the tensile strength value of the PVDF/ $/ \mathrm{Fe}_{3} \mathrm{O}_{4} @ \mathrm{PS}$ composite mats. Our present results showed higher tensile strength and elongation at break than those of the co-axial PS-polyurethane nanofiber reported in literature for oil sorption [47]. Here, the addition of PVDF nanofibers has significantly enhanced the overall mechanical properties of the PS mats, and even with the incorporation of $\mathrm{Fe}_{3} \mathrm{O}_{4} \mathrm{NPs}$ in/on PS, the composite mat still showed good mechanical property and enhanced elongation. However, the main reason for the incorporation of $\mathrm{Fe}_{3} \mathrm{O}_{4} \mathrm{NPs}$ in this study is to provide magnetic property to the composite mat for easier sorbent recovery.
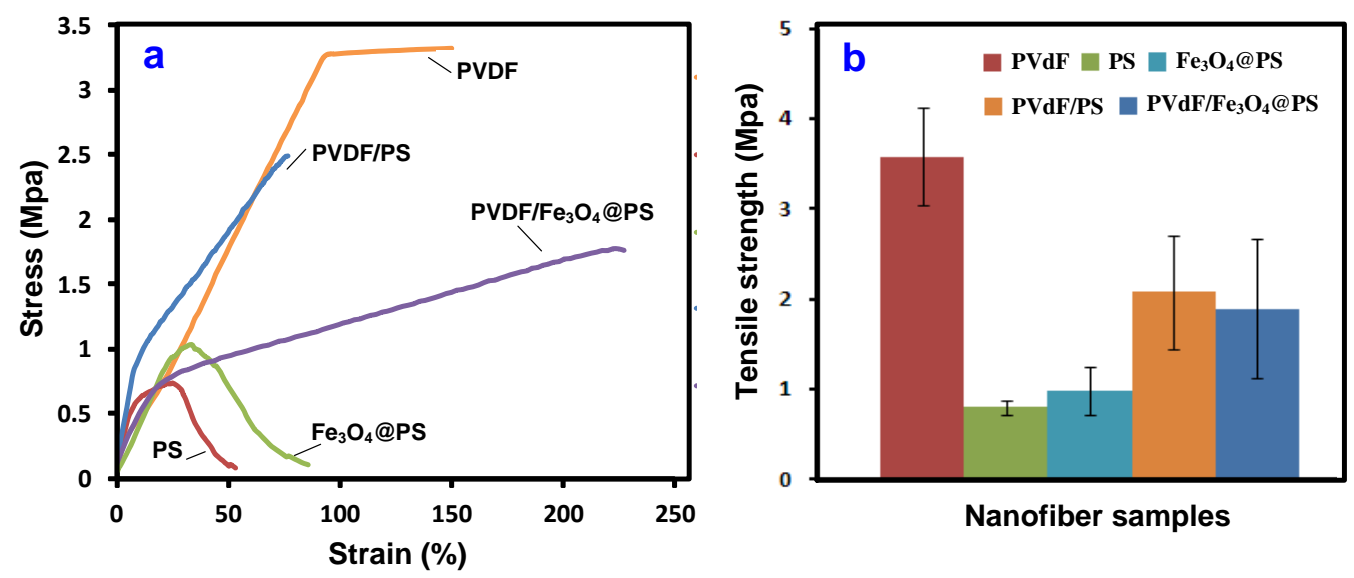

Fig. 7. (a) Typical stress-strain curves and (b) average tensile strength of the different fabricated mats in the present study.

\subsection{Oil sorption capacity in oil-in-water system}

The high capacity of oil adsorption mainly benefits from high porosity $[13,32]$. Since the CA tests already suggest the hydrophobicity of all the sorbent samples, the water sorption tests (not shown) furthermore confirmed their low adsorption ability to water. Though there is still tiny weight increase of the sorbent material after the sorption tests, it could have been due to the water attached on the surface or trapped on the space of the sorbent samples. 


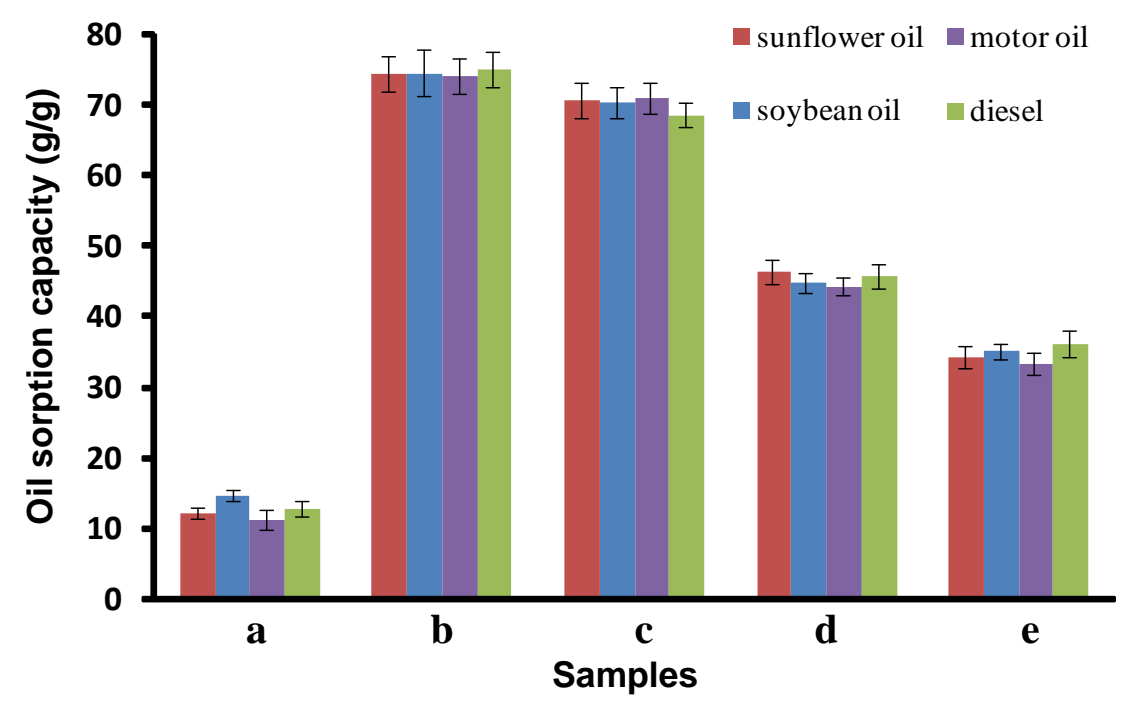

Fig. 8. Oil sorption capacity measurements of the of different fabricated mats: (a) PVDF, (b) PS, (c) Fe3O4/PS, (d) PVDF-PS, and (e) PVDF-Fe3O4/PS to 4 types of oil.

Figure 8 shows the oil sorption capacities of the different fabricated mats. Four oil types were utilized for the sorption test including sunflower oil, soybean oil, motor oil and diesel oil. The results showed that the PVDF nanofiber mat, which is innately hydrophobic, had low sorption capacity of 11$14 \mathrm{~g} / \mathrm{g}$ depending on the oil type. This is expected as PVDF does not exhibit high oleophilicity. On the other hand, the PS nanofibers showed excellent sorption ability up to 74-75 g/g, which is similar with those reported in previous studies [32]. The PS mat incorporated with iron oxide NPs showed similar sorption capacity as with the neat PS nanofibers, since there was no major change in fiber morphology and structure due to the presence of iron oxide NPs. When PS and PVDF were electrospun simultaneously, the resulting composite nanofibrous mats showed sorption capacity values that lie between the sorption capacities of both neat PVDF and neat PS mats. It should be noted that the fibrous mats were electrospun for a total of $4 \mathrm{ml}$ solution. This means that the composite bicomponent mats only contain approximately half the volume compared to the neat PS mat, and the other half is made of PVDF nanofibers. As a result, the oil sorption capacity of PVDF/PS and PVDF/Fe $\mathrm{O}_{4} @ \mathrm{PS}$ sorbents was 44-46 g/g and 35-36 g/g, respectively, which were lower than that obtained from neat PS mat. The PVDF nanofibers have smaller fiber diameter and larger surface area, which could have provided more interfiber voids in the composite mat, however, PVDF has low oleophilicity, thus it did not help much in the sorption ability. The presence of PVDF nanofibers though has drastically helped in improving the mechanical properties of the composite mat (see Fig. 7). The slight decrease of oil sorption ability after adding $\mathrm{Fe}_{3} \mathrm{O}_{4}$ NPs could be a result of $\mathrm{Fe}_{3} \mathrm{O}_{4}$ NPs possibly blocking and reducing the porosity of the mat. It is important to note that the sorption capacities of the composite mats here are still higher than those of commercial PP fibers, which have sorption capacities of 15-30 g/g [47]. The sorption capacity of all oil samples was similar with each other even when utilizing different 
fabricated mats. This could be attributed to the similar densities of the different oil samples at room temperature, and to the high porosities of all nanofiber mats. Previous studies [47] have found that the oil sorption capacity is mainly affected by the porosity or inter-fiber voids, and less likely by the internal porosity of the fibers themselves.

\section{Conclusions}

Oil spillage and oily water treatment have drawn more awareness and consideration nowadays because of its potential risk to the environment and the society. As a way of minimizing this oil pollution, sorbent materials with high surface area can be a potential candidate for oil cleanup. In this study, the oil sorption capacity of a composite sorbent material made of PVDF and $\mathrm{Fe}_{3} \mathrm{O}_{4} / \mathrm{PS}$ nanofibers fabricated by a one-step two-nozzle electrospinning process was investigated. All nanofiber mats showed highly porous structure. The mechanical properties of the composite mats containing both PS and PVDF were found to be significantly improved compared to the neat PS mat. The addition of $\mathrm{Fe}_{3} \mathrm{O}_{4}$ nanoparticles has provided magnetic property that helps for easier pick-up. Oil sorption test results showed adequate sorption capacity of 35-45 g/g for 4 types of oil, which are still higher than that of conventional PP fibers. The high interfiber porosity, interconnected pore structure, good mechanical property, possessing a magnetic property, and hydrophobic surface all contribute to the promising performance of the current composite sorbent. The results suggest the potential of the present magnetic composite nanofibrous mats for use in environmental remediation specifically on removal or separation of oil from the water surface.

\section{Acknowledgements}

This research was supported by a grant from the National Research Foundation (NRF) of Korea under the Ministry of Education, Science and Technology (Project no. 2013R1A2A2A04015484) and also was partially supported by the Industrial Strategic Technology Development Program (10044021).

\section{References}

1. Shen, H.C., Wang, J.X., Cheng, Y.T., Wei, Y.S., Zheng, J.W., Su, Y.F., et al., Risk and emergency treatment of marine oil spill: an overview, Environ Eng 2011;29(6):110-114, 85.

2. Kim, C.S., Cho, Y.K., Choi, B.J., Jung, K.T., You, S.H., Improving a prediction system for oil spills in the Yellow Sea: Effect of tides on subtidal flow, Mar Pollut Bull 2013;68(1-2):8592.

3. Tijing, L.D., Ruelo, M.T.G., Amarjargal, A., Pant, H.R., Park, C.-H., Kim, D.W., et al., Antibacterial and superhydrophilic electrospun polyurethane nanocomposite fibers containing tourmaline nanoparticles, Chem. Eng. J. 2012;197:41-48. 
4. Fritt-Rasmussen, J.Brandvik, P.J., Measuring ignitability for in situ burning of oil spills weathered under Arctic conditions: From laboratory studies to large-scale field experiments, Mar Pollut Bull 2011;62(8):1780-1785.

5. Castro, A., Iglesias, G., Carballo, R., Fraguela, J.A., Floating boom performance under waves and currents, J Hazard Mater 2010;174(1-3):226-235.

6. Prince, R.C., McFarlin, K.M., Butler, J.D., Febbo, E.J., Wang, F.C.Y., Nedwed, T.J., The primary biodegradation of dispersed crude oil in the sea, Chemosphere 2013;90(2):521-526.

7. Coelho, G., Clark, J., Aurand, D., Toxicity testing of dispersed oil requires adherence to standardized protocols to assess potential real world effects, Environ Pollut 2013;177:185188.

8. Al-Majed, A.A., Adebayo, A.R., Hossain, M.E., A sustainable approach to controlling oil spills, J Environ Manage 2012;113:213-227.

9. Ceylan, D., Dogu, S., Karacik, B., Yakan, S.D., Okay, O.S., Okay, O., Evaluation of butyl rubber as sorbent material for the removal of oil and polycyclic aromatic hydrocarbons from seawater, Environ Sci Technol 2009;43(10):3846-3852.

10. Wang, J., Zheng, Y., Kang, Y., Wang, A., Investigation of oil sorption capability of PBMA/SiO2 coated kapok fiber, Chem. Eng. J. 2013;223(0):632-637.

11. Ali, N., El-Harbawi, M., Jabal, A.A., Yin, C.Y., Characteristics and oil sorption effectiveness of kapok fibre, sugarcane bagasse and rice husks: oil removal suitability matrix, Environ Technol 2012;33(4):481-486.

12. Ge, B., Zhang, Z., Zhu, X., Men, X., Zhou, X., Xue, Q., A graphene coated cotton for oil/water separation, Compos. Sci. Technol. 2014;102(0):100-105.

13. Li, H., Liu, L.F., Yang, F.L., Hydrophobic modification of polyurethane foam for oil spill cleanup, Mar Pollut Bull 2012;64(8):1648-1653.

14. Lin, J.Y., Ding, B., Yang, J.M., Yu, J.Y., Sun, G., Subtle regulation of the micro- and nanostructures of electrospun polystyrene fibers and their application in oil absorption, Nanoscale 2012;4(1):176-182.

15. Duong, H.T.T.Burford, R.P., Effect of foam density, oil viscosity, and temperature on oil sorption behavior of polyurethane, J Appl Polym Sci 2006;99(1):360-367.

16. Chen, N.Pan, Q.M., Versatile fabrication of ultralight magnetic foams and application for oilwater separation, ACS Nano 2013;7(8):6875-6883.

17. Pavía-Sanders, A., Zhang, S., Flores, J.A., Sanders, J.E., Raymond, J.E., Wooley, K.L., Robust magnetic/polymer hybrid nanoparticles designed for crude oil entrapment and recovery in aqueous environments, ACS Nano 2013;7(9):7552-7561.

18. Wei, Q.F., Mather, R.R., Fotheringham, A.F., Oil removal from used sorbents using a biosurfactant, Biores Technol 2005;96(3):331-334. 
19. Ding, L., Li, Y., Jia, D., Deng, J.P., Yang, W.T., Beta-cyclodextrin-based oil-absorbents: Preparation, high oil absorbency and reusability, Carbohyd Polym 2011;83(4):1990-1996.

20. Tijing, L.D., Choi, J.-S., Lee, S., Kim, S.-H., Shon, H.K., Recent progress of membrane distillation using electrospun nanofibrous membrane, J Membrane Sci 2014;453:435-462.

21. Awal, A., Sain, M., Chowdhury, M., Preparation of cellulose-based nano-composite fibers by electrospinning and understanding the effect of processing parameters, Composites Part B: Engineering 2011;42(5):1220-1225.

22. Tijing, L.D., Woo, Y.C., Johir, M.A.H., Choi, J.-S., Shon, H.K., A novel dual-layer bicomponent electrospun nanofibrous membrane for desalination by direct contact membrane distillation, Chem. Eng. J. 2014;256:155-159.

23. Tijing, L.D., Park, C.-H., Choi, W.L., Ruelo, M.T.G., Amarjargal, A., Pant, H.R., et al., Characterization and mechanical performance comparison of multiwalled carbon nanotube/polyurethane composites fabricated by electrospinning and solution casting, Compos Part B: Eng 2013;44(1):613-619.

24. Kang, Y.O., Im, J.N., Park, W.H., Morphological and permeable properties of antibacterial double-layered composite nonwovens consisting of microfibers and nanofibers, Compos Part B: Eng 2015;75(0):256-263.

25. Tijing, L.D., Ruelo, M.T.G., Amarjargal, A., Pant, H.R., Park, C.H., Kim, C.S., One-step fabrication of antibacterial (silver nanoparticles/poly(ethylene oxide)) - Polyurethane bicomponent hybrid nanofibrous mat by dual-spinneret electrospinning, Mater Chem Phys 2012;134(2-3):557-561.

26. Tijing, L.D., Choi, W., Jiang, Z., Amarjargal, A., Park, C.-H., Pant, H.R., et al., Two-nozzle electrospinning of (MWNT/PU)/PU nanofibrous composite mat with improved mechanical and thermal properties, Curr Appl Phys 2013;13(7):1247-1255.

27. Zhu, H., Qiu, S., Jiang, W., Wu, D., Zhang, C., Evaluation of electrospun polyvinyl chloride/polystyrene fibers as sorbent materials for oil spill cleanup, Environ Sci Technol 2011;45(10):4527-4531.

28. Saghafi, H., Brugo, T., Minak, G., Zucchelli, A., The effect of PVDF nanofibers on mode-I fracture toughness of composite materials, Compos Part B: Eng 2015;72(0):213-216.

29. Chen, Q., Zhao, Y., Zhou, Z., Rahman, A., Wu, X.-F., Wu, W., et al., Fabrication and mechanical properties of hybrid multi-scale epoxy composites reinforced with conventional carbon fiber fabrics surface-attached with electrospun carbon nanofiber mats, Compos Part B: Eng 2013;44(1):1-7.

30. Ribeiro, S., Costa, P., Ribeiro, C., Sencadas, V., Botelho, G., Lanceros-Méndez, S., Electrospun styrene-butadiene-styrene elastomer copolymers for tissue engineering applications: Effect of butadiene/styrene ratio, block structure, hydrogenation and carbon 
nanotube loading on physical properties and cytotoxicity, Compos Part B: Eng 2014;67(0):30-38.

31. Shen, J., Li, Z., Wu, Y.-n., Zhang, B., Li, F., Dendrimer-based preparation of mesoporous alumina nanofibers by electrospinning and their application in dye adsorption, Chem. Eng. J. 2015;264(0):48-55.

32. Lin, J.Y., Shang, Y.W., Ding, B., Yang, J.M., Yu, J.Y., Al-Deyab, S.S., Nanoporous polystyrene fibers for oil spill cleanup, Mar Pollut Bull 2012;64(2):347-352.

33. Liao, Y., Wang, R., Tian, M., Qiu, C.Q., Fane, A.G., Fabrication of polyvinylidene fluoride (PVDF) nanofiber membranes by electro-spinning for direct contact membrane distillation, $\mathrm{J}$ Mem Sci 2013;425:30-39.

34. Lalia, B.S., Guillen-Burrieza, E., Arafat, H.A., Hashaikeh, R., Fabrication and characterization of polyvinylidenefluoride-co-hexafluoropropylene (PVDF-HFP) electrospun membranes for direct contact membrane distillation, J Mem Sci 2013;428:104-115.

35. Jain, T.K., Reddy, M.K., Morales, M.A., Leslie-Pelecky, D.L., Labhasetwar, V., Biodistribution, clearance, and biocompatibility of iron oxide magnetic nanoparticles in rats, Mol Pharmaceut 2008;5(2):316-327.

36. Hakami, O., Zhang, Y., Banks, C.J., Thiol-functionalised mesoporous silica-coated magnetite nanoparticles for high efficiency removal and recovery of $\mathrm{Hg}$ from water, Water Res 2012;46(12):3913-3922.

37. Yuan, X.P.Chung, T.C.M., Novel Solution to Oil Spill Recovery: Using thermodegradable polyolefin oil superabsorbent polymer (oil-SAP), Energ Fuel 2012;26(8):4896-4902.

38. Bhardwaj, N.Kundu, S.C., Electrospinning: A fascinating fiber fabrication technique, Biotechnol Adv 2010;28(3):325-347.

39. Lee, M.W., An, S., Joshi, B., Latthe, S.S., Yoon, S.S., Highly efficient wettability control via three-dimensional (3D) suspension of titania nanoparticles in polystyrene nanofibers, ACS Appl Mater Interf 2013;5(4):1232-1239.

40. Wang, X.F., Ding, B., Yu, J.Y., Wang, M.R., Engineering biomimetic superhydrophobic surfaces of electrospun nanomaterials, Nano Today 2011;6(5):510-530.

41. Wu, N., Cao, Q., Wang, X.Y., Chen, Q.Q., Study of a novel porous gel polymer electrolyte based on TPU/PVdF by electrospinning technique, Solid State Ionics 2011;203(1):42-46.

42. Na, H.N., Chen, P., Wong, S.C., Hague, S., Li, Q., Fabrication of PVDF/PVA microtubules by coaxial electrospinning, Polymer 2012;53(13):2736-2743.

43. Granitzer, P., Rumpf, K., Venkatesan, M., Roca, A.G., Cabrera, L., Morales, M.P., et al., Magnetic study of $\mathrm{Fe}_{3} \mathrm{O}_{4}$ Nanoparticles incorporated within mesoporous silicon, J. Electrochem. Soc. 2010;157(7):K145-K151.

44. Roach, P., Shirtcliffe, N.J., Newton, M.I., Progess in superhydrophobic surface development, Soft Matter 2008;4(2):224-240. 
45. Ying, Z., Jing, C.Z., Jin, Z., Yong, M.Z., Lin, F., Lei, J., Multifunctional carbon nanofibers with conductive, magnetic and superhydrophobic properties, Chemphyschem 2006;7(2):336341.

46. Avila, A.F., Munhoz, V.C., de Oliveira, A.M., Santos, M.C.G., Lacerda, G.R.B.S., Gonçalves, C.P., Nano-based systems for oil spills control and cleanup, J. Hazard. Mater. 2014;272(0):20-27.

47. Lin, J., Tian, F., Shang, Y., Wang, F., Ding, B., Yu, J., et al., Co-axial electrospun polystyrene/polyurethane fibres for oil collection from water surface, Nanoscale 2013;5(7):2745-2755. 


\section{Figure and table captions}

Fig. 1. Schematic illustration of the present multi-nozzle electrospinning set-up.

Fig. 2. FE-SEM images and corresponding fiber distributions (insets) of the electrospun fibers and composites: (a) PVDF, (b) PS. (c) $\mathrm{Fe}_{3} \mathrm{O}_{4} @ \mathrm{PS}$, (d) PVDF/PS, and (e) PVDF/Fe $\mathrm{O}_{4} @$ PS. The single dotted circles indicate the presence of $\mathrm{Fe}_{3} \mathrm{O}_{4}$ NPs. The double dotted circles indicate the presence of beads on string.

Fig. 3. Optical images of the fabricated mats: (a)PVDF, (b)PS. (c) $\mathrm{Fe}_{3} \mathrm{O}_{4} @ \mathrm{PS},(\mathrm{d}) \mathrm{PVDF} / \mathrm{PS},(\mathrm{e})$ $\mathrm{PVDF} / \mathrm{Fe}_{3} \mathrm{O}_{4} @ \mathrm{PS}$, and (f) magnetic composite mat when exposed to an external magnet.

Fig. 4. TEM images of electrospun $\mathrm{Fe}_{3} \mathrm{O}_{4} @ \mathrm{PS}$ nanofiber at different locations of the nanofiber mat.

Fig. 5. FT-IR spectra of different fabricated mats: (a) PVDF, (b) PS, (c) $\mathrm{Fe}_{3} \mathrm{O}_{4} / \mathrm{PS}$, (d) PVDF-PS, and (e) $\mathrm{PVDF}-\mathrm{Fe}_{3} \mathrm{O}_{4} / \mathrm{PS}$.

Fig. 6. Contact angles of different fabricated mats: (a) PVDF, (b) PS, (c) $\mathrm{Fe}_{3} \mathrm{O}_{4} / \mathrm{PS}$, (d) PVDF-PS, and (e) $\mathrm{PVDF}-\mathrm{Fe}_{3} \mathrm{O}_{4} / \mathrm{PS}$.

Fig. 7. (a) Typical stress-strain curves and (b) average tensile strength of the different fabricated mats in the present study.

Fig. 8. Oil sorption capacity measurements of the of different fabricated mats: (a) PVDF, (b) PS, (c) $\mathrm{Fe}_{3} \mathrm{O}_{4} / \mathrm{PS}$, (d) PVDF-PS, and (e) PVDF-Fe $\mathrm{O}_{4} / \mathrm{PS}$ to 4 types of oil

Table 1. Properties of the oils used in the present study.

Table 2. Electrospinning parameters and the corresponding names of the fabricated mats. 